\title{
THE PROBLEM OF DIFFERENTIATION OF HYPERELLIPTIC FUNCTIONS
}

\author{
ELENA YU. BUNKOVA
}

\begin{abstract}
In this work we describe a construction that leads to an explicit solution of the problem of differentiation of hyperelliptic functions. A classical genus $g=1$ example of such a solution is the result of F. G. Frobenius and L. Stickelberger [1].

Our method follows the works [2] and [3] that led to constructions of explicit solutions of the problem for genus $g=2$ and $g=3$.
\end{abstract}

\section{INTRODUCTION}

We consider meromorphic functions $f$ in $\mathbb{C}^{g}$. A vector $\omega \in \mathbb{C}^{g}$ is called a period for $f$ if $f(z+\omega)=f(z)$ for any $z \in \mathbb{C}^{g}$. If the periods of $f$ form a lattice $\Gamma$ of rank $2 g$ in $\mathbb{C}^{g}$, then $f$ is called an Abelian function. We say that an Abelian function is a meromorphic function on the complex torus $T^{g}=\mathbb{C}^{g} / \Gamma$. We denote the coordinates in $\mathbb{C}^{g}$ by $\left(z_{1}, z_{3}, \ldots, z_{2 g-1}\right)$.

Let us consider hyperelliptic curves of genus $g$ in the model

$$
\mathcal{V}_{\lambda}=\left\{(X, Y) \in \mathbb{C}^{2}: Y^{2}=X^{2 g+1}+\lambda_{4} X^{2 g-1}+\lambda_{6} X^{2 g-2}+\ldots+\lambda_{4 g} X+\lambda_{4 g+2}\right\}
$$

Such a curve depends on the parameters $\lambda=\left(\lambda_{4}, \lambda_{6}, \ldots, \lambda_{4 g}, \lambda_{4 g+2}\right) \in \mathbb{C}^{2 g}$.

Denote by $\mathcal{B} \subset \mathbb{C}^{2 g}$ the subspace of parameters such that $\mathcal{V}_{\lambda}$ is non-singular for $\lambda \in \mathcal{B}$. We have $\mathcal{B}=\mathbb{C}^{2 g} \backslash \Sigma$ where $\Sigma$ is the discriminant curve.

A hyperelliptic function of genus $g$ (see [2, 4]) is a meromorphic function in $\mathbb{C}^{g} \times \mathcal{B}$, such that for each $\lambda \in \mathcal{B}$ it's restriction to $\mathbb{C}^{g} \times \lambda$ is Abelian with $T^{g}$ the Jacobian $\mathcal{J}_{\lambda}$ of $\mathcal{V}_{\lambda}$. We denote the field of hyperelliptic functions of genus $g$ by $\mathcal{F}$. See [4] for it's properties.

Let $\mathcal{U}$ be the space of the fiber bundle $\pi: \mathcal{U} \rightarrow \mathcal{B}$ with fiber over $\lambda \in \mathcal{B}$ the Jacobian $\mathcal{J}_{\lambda}$ of the curve $\mathcal{V}_{\lambda}$. Thus, a hyperelliptic function is a meromorphic function in $\mathcal{U}$. According to Dubrovin-Novikov theorem [5], there is a birational isomorphism between $\mathcal{U}$ and the complex linear space $\mathbb{C}^{3 g}$.

Problem 1.1 ([4]). For each g describe the Lie algebra Der $\mathcal{F}$ of differentiations of $\mathcal{F}$, that is find $3 g$ independent differential operators $\mathcal{L}$ such that $\mathcal{L} \mathcal{F} \subset \mathcal{F}$.

In case $g=1$ the solution of this problem is classical [1. A method for solving this problem in a general case was presented in [6, 7]. A good overview of this approach is given in [4]. It turned out that it is hard to follow this method to obtain explicit answers.

Explicit solutions to this problem for $g=2$ and $g=3$ were first found in [2] and [3]. This works allow us to present a general method that is useful for any genus. Here we describe the general construction of this method.

We use the theory of hyperelliptic Kleinian functions (see [8, 9, 10, 11], and [12] for elliptic functions). Take the coordinates $(z, \lambda)=\left(z_{1}, z_{3}, \ldots, z_{2 g-1}, \lambda_{4}, \lambda_{6}, \ldots, \lambda_{4 g}, \lambda_{4 g+2}\right)$

Key words and phrases. Abelian functions, elliptic functions, Jacobians, hyperelliptic curves, hyperelliptic functions, Lie algebra of derivations, polynomial vector fields.

Supported in part by Young Russian Mathematics award and the RFBR project 17-01-00366 A. 
in $\mathbb{C}^{g} \times \mathcal{B} \subset \mathbb{C}^{3 g}$. Let $\sigma(z, \lambda)$ be the hyperelliptic sigma function (or elliptic sigma function in genus $g=1$ case). We denote $\partial_{k}=\frac{\partial}{\partial z_{k}}$. Following [2, 3, 4], we use the notation

$$
\zeta_{k}=\partial_{k} \ln \sigma(z, \lambda), \quad \wp_{i ; k_{1}, \ldots, k_{n}}=-\partial_{1}^{i} \partial_{k_{1}} \cdots \partial_{k_{n}} \ln \sigma(z, \lambda)
$$

where $n \geqslant 0, i+n \geqslant 2, k_{s} \in\{1,3, \ldots, 2 g-1\}$. In the case $n=0$ we will skip the semicolon. Note that our notation for the variables $z_{k}$ differs from the one in [9, 10, 11] as $u_{i}=z_{2 g+1-2 i}$. The functions $\wp_{i ; k_{1}, \ldots, k_{n}}$ provide us with examples of hyperelliptic functions.

A key to our approach to the problem is the following theorem:

Theorem $1.2([9])$. For $i, k \in\{1,3, \ldots, 2 g-1\}$ we have the relations

$$
\begin{aligned}
\wp_{3 ; i}= & 6 \wp_{2} \wp_{1 ; i}+6 \wp_{1 ; i+2}-2 \wp_{0 ; 3, i}+2 \lambda_{4} \delta_{i, 1}, \\
\wp_{2 ; i} \wp_{2 ; k}= & \left.4 \wp_{2} \wp_{1 ; i} \wp_{1 ; k}+\wp_{1 ; k} \wp_{1 ; i+2}+\wp_{1 ; i} \wp_{1 ; k+2}+\wp_{0 ; k+2, i+2}\right)- \\
& -2\left(\wp_{1 ; i} \wp_{0 ; 3, k}+\wp_{1 ; k} \wp_{0 ; 3, i}+\wp_{0 ; k, i+4}+\wp_{0 ; i, k+4}\right)+ \\
& +2 \lambda_{4}\left(\delta_{i, 1} \wp_{1 ; k}+\delta_{k, 1} \wp_{1 ; i}\right)+2 \lambda_{i+k+4}\left(2 \delta_{i, k}+\delta_{k, i-2}+\delta_{i, k-2}\right) .
\end{aligned}
$$

Proof. In [9] we have formulas (4.1) and (4.8). Using the notation (2) we get (3) from (4.1) and (4) from (4.8).

\section{THE PROBLEM FOR POLYNOMIAL VECTOR FIELDS}

The work [13] constructs the theory of polynomial Lie algebras. Here we describe its connection with Problem 1.1.

Consider the complex space $\mathbb{C}^{3 g}$ with coordinates $x=\left(x_{i, j}\right)$, where $i \in\{1,2,3\}$, $j \in\{1,3, \ldots, 2 g-1\}$. We define the map $\varphi: \mathcal{U} \rightarrow \mathbb{C}^{3 g}$ by

$$
\varphi:(z, \lambda) \mapsto\left(x_{i, j}\right)=\left(\wp_{i ; j}(z, \lambda)\right) .
$$

This map has the following property, proposed by V. M. Buchstaber (see [2]):

Theorem 2.1. The functions $\varphi^{*}\left(x_{i, j}\right)$ give a set of generators of $\mathcal{F}$.

Proof. We show that the functions $\wp_{i ; j}(z, \lambda)$, where $i \in\{1,2,3\}, j \in\{1,3, \ldots, 2 g-1\}$, give a set of generators of $\mathcal{F}$.

We use a fundamental result from the theory of hyperelliptic Abelian functions (see [11, Chapter 5]): Any hyperelliptic function can be presented as a rational function in $\wp_{1 ; k}$ and $\wp_{2 ; k}$, where $k \in\{1,3, \ldots, 2 g-1\}$. Theorem 1.2 gives a set of relations between the derivatives of this functions.

Now by [3, Corollary 5.2], the functions $\left(\varphi^{*}\left(x_{i, j}\right), \varphi^{*}\left(w_{k, l}\right), \varphi^{*}\left(\lambda_{s}\right)\right)$ in the notation of this Corollary give a set of generators of $\mathcal{F}$. By [3, Theorem 5.3] we obtain the claim of Theorem 2.1.

Another property of $\varphi$ follows form [3, Corollary 5.5]. For each $g$ a there is polynomial map $p: \mathbb{C}^{3 g} \rightarrow \mathbb{C}^{2 g}$, such that we get the diagram

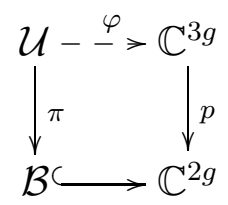

Here $\mathcal{B} \subset \mathbb{C}^{2 g}$ is the inclusion like in section 1, with coordinates $\lambda$ in $\mathbb{C}^{2 g}$.

We note that the proof of [3, Theorem 5.3] gives a construction to obtain the polynomial maps $p$ explicitly. Examples of this maps for $g=1,2,3$ are given in [3]. The work [4, Theorem 3.2] claims that this polynomial maps are of degree at most 3. 
We refer the reader to [13] for the theory of polynomial Lie algebras. Denote the ring of polynomials in $\lambda \in \mathbb{C}^{2 g}$ by $\mathcal{P}$. Let us consider the polynomial map $p: \mathbb{C}^{3 g} \rightarrow \mathbb{C}^{2 g}$. A vector field $\mathcal{L}$ in $\mathbb{C}^{3 g}$ will be called projectable for $p$ if there exists a vector field $L$ in $\mathbb{C}^{2 g}$ such that

$$
\mathcal{L}\left(p^{*} f\right)=p^{*} L(f) \quad \text { for any } \quad f \in \mathcal{P} .
$$

The vector field $L$ will be called the pushforward of $\mathcal{L}$. A corollary of this definition is that for a projectable vector field $\mathcal{L}$ we have $\mathcal{L}\left(p^{*} \mathcal{P}\right) \subset p^{*} \mathcal{P}$.

Problem 2.2 ([3, Problem 6.1]). Find $3 g$ polynomial vector fields in $\mathbb{C}^{3 g}$ projectable for $p: \mathbb{C}^{3 g} \rightarrow \mathbb{C}^{2 g}$ and independent at any point in $p^{-1}(\mathcal{B})$. Construct their polynomial Lie algebra.

The connection of this problem to Problem 1.1 is straightforward. Given a solution to Problem 2.2 for each of the $3 g$ vector fields $\mathcal{L}_{k}$ with pushforwards $L_{k}$ we will restore the vector fields $\mathcal{L}_{k}$ projectable for $\pi$ with pushforwards $L_{k}$ and such that $\mathcal{L}_{k}\left(\varphi^{*} x_{i, j}\right)=$ $\varphi^{*} \mathcal{L}_{k}\left(x_{i, j}\right)$ for the coordinate functions $x_{i, j}$ in $\mathbb{C}^{3 g}$. As $\varphi^{*} x_{i, j}$ are the generators of $\mathcal{F}$ and $\mathcal{L}_{k}\left(x_{i, j}\right)$ is a polynomial in $x_{i, j}$, this gives $\mathcal{L}_{k}\left(\varphi^{*} x_{i, j}\right) \in \mathcal{F}$ and $\mathcal{L}_{k} \in \operatorname{Der} \mathcal{F}$.

The plan to solve Problem 2.2 is the following. For each $g$ :

- Find the "odd polynomial vector fields", i.e. the $g$ independant polynomial vector fields $\mathcal{L}_{1}, \mathcal{L}_{3}, \ldots, \mathcal{L}_{2 g-1}$ projectable for $p$ with zero pushforward.

- Define $2 g$ independant polynomial vector fields $L_{0}, L_{2}, L_{4}, \ldots, L_{4 g-2}$ in $\mathcal{B}$.

- Find the "even polynomial vector fields", i.e. the $2 g$ polynomial vector fields $\mathcal{L}_{0}, \mathcal{L}_{2}, \mathcal{L}_{4}, \ldots, \mathcal{L}_{4 g-2}$ projectable for $p$ with pushforwards $L_{0}, L_{2}, L_{4}, \ldots, L_{4 g-2}$.

- Construct their polynomial Lie algebra.

We will do this steps in the following sections. In the last section we give the explicit solutions for problem 1.1 that can be constucted by this method (see [3]).

\section{ODD POLYNOMIAL VECTOR FIELDS}

Lemma 3.1 ([3, Lemma 6.2 and Lemma 6.3]). We have

$$
\mathcal{L}_{1}=\sum_{j} x_{2, j} \frac{\partial}{\partial x_{1, j}}+x_{3, j} \frac{\partial}{\partial x_{2, j}}+4\left(2 x_{2} x_{2, j}+x_{3} x_{1, j}+x_{2, j+2}\right) \frac{\partial}{\partial x_{3, j}}
$$

where $x_{2,2 g+1}=0$. For $s=3,5, \ldots, 2 g-1$ we have

$$
\begin{aligned}
\mathcal{L}_{s}=x_{2, s} & \frac{\partial}{\partial x_{2}}+x_{3, s} \frac{\partial}{\partial x_{3}}+\mathcal{L}_{1}\left(x_{3, s}\right) \frac{\partial}{\partial x_{4}}+ \\
& +\sum_{k=1}^{g-1} z_{1, s, 2 k+1} \frac{\partial}{\partial x_{1,2 k+1}}+\mathcal{L}_{1}\left(z_{1, s, 2 k+1}\right) \frac{\partial}{\partial x_{2,2 k+1}}+\mathcal{L}_{1}\left(\mathcal{L}_{1}\left(z_{1, s, 2 k+1}\right)\right) \frac{\partial}{\partial x_{3,2 k+1}} .
\end{aligned}
$$

for some $y_{1, s, 2 k+1}=\mathcal{L}_{s}\left(x_{1,2 k+1}\right)$.

This lemma determines the odd polynomial vector fields given the value $\mathcal{L}_{s}\left(x_{1,2 k+1}\right)$. For this value we use the construction of Korteweg-de Vries hierarchy [10, Section 4.4]. 
The Korteweg-de Vries equation

$$
u_{t}=6 u u_{x}-u_{x x x}
$$

for $x=z_{1},-4 t=z_{3}, \Phi_{2}=\frac{1}{2} u, \Phi_{4}=-\frac{3}{2} \Phi_{2}^{2}+\frac{1}{4} \partial_{1} \Phi_{2}$ takes the form

$$
\partial_{3} \Phi_{2}=\partial_{1} \Phi_{4}
$$

It is the first equation of the Korteweg-de Vries hierarchy, which is an infinite system of differential equations

$$
\partial_{2 k-1} \Phi_{2}=\partial_{1} \Phi_{2 k}, \quad k=2,3,4, \ldots
$$

where

$$
\partial_{1} \Phi_{2 k+2}=\mathcal{R} \partial_{1} \Phi_{2 k} \quad \text { and } \quad \mathcal{R}=\frac{1}{4} \partial_{1}^{2}-2 \Phi_{2}-\Phi_{2}^{\prime} \partial_{1}^{-1}
$$

Theorem 3.2 ([10, Theorem 4.12]). The function $u=2 \wp_{2}(z)$ is a g-gap solution of the Korteweg-de Vries system.

This gives us a system of equations

$$
\mathcal{L}_{s}\left(x_{2}\right)=\mathcal{L}_{1} \Phi_{s}\left(x_{2}\right)
$$

with differential polynomials $\Phi_{s}$. Thus in Lemma 3.1 we have

$$
\mathcal{L}_{s}\left(x_{1,2 k+1}\right)=\mathcal{L}_{s}\left(\Phi_{2 k}\left(x_{2}\right)\right) .
$$

This determines $y_{1, s, 2 k+1}$.

\section{EVEN POLYNOMIAL VECTOR FIELDS}

First we define the polynomial vector fields in $\mathcal{B}$. Recall $\mathcal{B}=\mathbb{C}^{2 g} \backslash \Sigma$ where $\Sigma$ is the discriminant curve.

For the vector fields $L_{0}, L_{2}, L_{4}, \ldots, L_{4 g-2}$ in $\mathcal{B}$ we take the vector fields tangent to $\Sigma$, that are obtained from the convolution of invariants of the group $A_{\mu}$, see the construction by D.B.Fuchs in [14, Section 4]. See also [13] and [15].

We consider $\mathbb{C}^{2 g}$ with coordinates $\left(\lambda_{4}, \lambda_{6}, \ldots, \lambda_{4 g}, \lambda_{4 g+2}\right)$ and set $\lambda_{s}=0$ for every $s \notin\{4,6, \ldots, 4 g, 4 g+2\}$. For $k, m \in\{1,2, \ldots, 2 g\}, k \leqslant m$ set

$$
T_{2 k, 2 m}=2(k+m) \lambda_{2 k+2 m}+\sum_{s=2}^{k-1} 2(k+m-2 s) \lambda_{2 s} \lambda_{2 k+2 m-2 s}-\frac{2 k(2 g-m+1)}{2 g+1} \lambda_{2 k} \lambda_{2 m},
$$

and for $k>m$ set $T_{2 k, 2 m}=T_{2 m, 2 k}$. For $k=0,1,2, \ldots, 2 g-1$ we have the vector fields

$$
L_{2 k}=\sum_{s=2}^{2 g+1} T_{2 k+2,2 s-2} \frac{\partial}{\partial \lambda_{2 s}} .
$$

The expressions (8) give polynomial vector fields tangent to the discriminant curve.

Now we need to find polynomial vector fields $\mathcal{L}_{2 k}$ projectable for $p$ with pushforwards $L_{2 k}$. The vector field $\mathcal{L}_{0}$ is the Euler vector field on $\mathbb{C}^{3 g}$, we have

$$
\mathcal{L}_{0}=\sum_{j}(j+1) x_{1, j} \frac{\partial}{\partial x_{1, j}}+(j+2) x_{2, j} \frac{\partial}{\partial x_{2, j}}+(j+3) x_{3, j} \frac{\partial}{\partial x_{3, j}} .
$$


All the other vector fields are determined using the condition on the polynomial Lie algebra

$$
\left(\begin{array}{c}
{\left[\mathcal{L}_{1}, \mathcal{L}_{0}\right]} \\
{\left[\mathcal{L}_{1}, \mathcal{L}_{2}\right]} \\
{\left[\mathcal{L}_{1}, \mathcal{L}_{4}\right]} \\
{\left[\mathcal{L}_{1}, \mathcal{L}_{6}\right]} \\
\vdots \\
{\left[\mathcal{L}_{1}, \mathcal{L}_{4 g-4}\right]} \\
{\left[\mathcal{L}_{1}, \mathcal{L}_{4 g-2}\right]}
\end{array}\right)=\left(\begin{array}{ccccc}
-1 & 0 & 0 & \ldots & 0 \\
x_{1,1} & -1 & 0 & \ldots & 0 \\
x_{1,3} & x_{1,1} & -1 & \ldots & 0 \\
x_{1,5} & x_{1,3} & x_{1,1} & \ldots & 0 \\
\ddots & \ddots & \ddots & \ddots & \ddots \\
0 & 0 & \ldots & x_{1,2 g-1} & x_{1,2 g-3} \\
0 & 0 & \ldots & 0 & x_{1,2 g-1}
\end{array}\right)\left(\begin{array}{c}
\mathcal{L}_{1} \\
\mathcal{L}_{3} \\
\vdots \\
\mathcal{L}_{2 g-1}
\end{array}\right)
$$

A demonstration of this method for genus $g=4$ will follow in our upcoming works.

\section{Explicit solutions of the Problem of Differentiation of Hyperelliptic FunCtions}

5.1. Genus 1. See [1]. The generators of the $\mathcal{F}$-module Der $\mathcal{F}$ are

$$
\mathcal{L}_{0}=L_{0}-z_{1} \partial_{1}, \quad \mathcal{L}_{1}=\partial_{1}, \quad \mathcal{L}_{2}=L_{2}-\zeta_{1} \partial_{1},
$$

Their Lie algebra is $\left[\mathcal{L}_{0}, \mathcal{L}_{1}\right]=\mathcal{L}_{1},\left[\mathcal{L}_{0}, \mathcal{L}_{2}\right]=2 \mathcal{L}_{2},\left[\mathcal{L}_{1}, \mathcal{L}_{2}\right]=\wp_{2} \mathcal{L}_{1}$.

5.2. Genus 2. The generators of the $\mathcal{F}$-module Der $\mathcal{F}$ are (see [2, Theorem 29]):

$$
\begin{aligned}
\mathcal{L}_{0} & =L_{0}-z_{1} \partial_{1}-3 z_{3} \partial_{3}, & \mathcal{L}_{2} & =L_{2}+\left(-\zeta_{1}+\frac{4}{5} \lambda_{4} z_{3}\right) \partial_{1}-z_{1} \partial_{3}, \\
\mathcal{L}_{1} & =\partial_{1}, & \mathcal{L}_{4} & =L_{4}+\left(-\zeta_{3}+\frac{6}{5} \lambda_{6} z_{3}\right) \partial_{1}-\left(\zeta_{1}+\lambda_{4} z_{3}\right) \partial_{3}, \\
\mathcal{L}_{3} & =\partial_{3}, & \mathcal{L}_{6} & =L_{6}+\frac{3}{5} \lambda_{8} z_{3} \partial_{1}-\zeta_{3} \partial_{3} .
\end{aligned}
$$

Their Lie algebra can be found in [2, Theorem 32].

5.3. Genus 3. The generators of the $\mathcal{F}$-module Der $\mathcal{F}$ are (see [3, Theorem 10.1]):

$$
\begin{aligned}
\mathcal{L}_{1} & =\partial_{1}, \quad \mathcal{L}_{3}=\partial_{3}, \quad \mathcal{L}_{5}=\partial_{5} \\
\mathcal{L}_{0} & =L_{0}-z_{1} \partial_{1}-3 z_{3} \partial_{3}-5 z_{5} \partial_{5} \\
\mathcal{L}_{2} & =L_{2}-\left(\zeta_{1}-\frac{8}{7} \lambda_{4} z_{3}\right) \partial_{1}-\left(z_{1}-\frac{4}{7} \lambda_{4} z_{5}\right) \partial_{3}-3 z_{3} \partial_{5} \\
\mathcal{L}_{4} & =L_{4}-\left(\zeta_{3}-\frac{12}{7} \lambda_{6} z_{3}\right) \partial_{1}-\left(\zeta_{1}+\lambda_{4} z_{3}-\frac{6}{7} \lambda_{6} z_{5}\right) \partial_{3}-\left(z_{1}+3 \lambda_{4} z_{5}\right) \partial_{5}, \\
\mathcal{L}_{6} & =L_{6}-\left(\zeta_{5}-\frac{9}{7} \lambda_{8} z_{3}\right) \partial_{1}-\left(\zeta_{3}-\frac{8}{7} \lambda_{8} z_{5}\right) \partial_{3}-\left(\zeta_{1}+\lambda_{4} z_{3}+2 \lambda_{6} z_{5}\right) \partial_{5}, \\
\mathcal{L}_{8} & =L_{8}+\left(\frac{6}{7} \lambda_{10} z_{3}-\lambda_{12} z_{5}\right) \partial_{1}-\left(\zeta_{5}-\frac{10}{7} \lambda_{10} z_{5}\right) \partial_{3}-\left(\zeta_{3}+\lambda_{8} z_{5}\right) \partial_{5}, \\
\mathcal{L}_{10} & =L_{10}+\left(\frac{3}{7} \lambda_{12} z_{3}-2 \lambda_{14} z_{5}\right) \partial_{1}+\frac{5}{7} \lambda_{12} z_{5} \partial_{3}-\zeta_{5} \partial_{5} .
\end{aligned}
$$

Their Lie algebra can be found in [3, Corollary 10.2]. 


\section{REFERENCES}

[1] F. G. Frobenius, L. Stickelberger, Über die Differentiation der elliptischen Functionen nach den Perioden und Invarianten, J. Reine Angew. Math., 92 (1882), 311-337.

[2] V. M. Buchstaber, Polynomial dynamical systems and Korteweg-de Vries equation, Proc. Steklov Inst. Math., 294 (2016), 176-200.

[3] E. Yu. Bunkova, Differentiation of genus 3 hyperelliptic functions, European Journal of Mathematics, 4:1 (2018), 93-112, arXiv: 1703.03947

[4] V. Buchstaber, V. Enolski, D. Leykin, Multi-variable sigma-functions: old and new results, 2018, arXiv: 1810.11079 .

[5] B. A. Dubrovin, S. P. Novikov, A periodic problem for the Korteweg-de Vries and Sturm-Liouville equations. Their connection with algebraic geometry. (Russian) Dokl. Akad. Nauk SSSR, 219:3, 1974, 531-534.

[6] V. M. Buchstaber, D. V. Leikin, Differentiation of Abelian functions with respect to parameters, Russian Math. Surveys, 62:4 (2007), 787-789.

[7] V. M. Buchstaber, D. V. Leikin, Solution of the Problem of Differentiation of Abelian Functions over Parameters for Families of (n, s)-Curves, Funct. Anal. Appl., 42:4, 2008, 268-278.

[8] H. F. Baker, On the hyperelliptic sigma functions, Amer. Journ. Math. 20, 1898, 301-384.

[9] V. M. Buchstaber, V. Z. Enolskii, D. V. Leikin, Kleinian functions, hyperelliptic Jacobians and applications, Reviews in Mathematics and Math. Physics, 10:2, Gordon and Breach, London, 1997, 3-120.

[10] V. M. Buchstaber, V. Z. Enolskii, D. V. Leikin, Hyperelliptic Kleinian functions and applications, "Solitons, Geometry and Topology: On the Crossroad", Adv. Math. Sci., AMS Transl., 179:2, Providence, RI, 1997, 1-34.

[11] V. M. Buchstaber, V. Z. Enolskii, D. V. Leikin, Multi-Dimensional Sigma-Functions, arXiv: 1208.0990, 2012, 267 pp.

[12] E. T. Whittaker, G. N. Watson, A Course of Modern Analysis, Reprint of 4th (1927) ed., Vol 2. Transcendental functions, Cambridge Univ. Press, Cambridge, 1996.

[13] V. M. Buchstaber, D. V. Leikin, Polynomial Lie Algebras, Funct. Anal. Appl., 36:4 (2002), 267-280.

[14] V. I. Arnold, Singularities of Caustics and Wave Fronts, Mathematics and its Applications, vol. 62, Kluwer Academic Publisher Group, Dordrecht, 1990.

[15] V. M. Buchstaber, A. V. Mikhailov, Infinite-Dimensional Lie Algebras Determined by the Space of Symmetric Squares of Hyperelliptic Curves, Functional Analysis and Its Applications, Vol. 51, No. 1, 2017.

Steklov Mathematical Institute of Russian Academy of Sciences, 8 Gubkina St., Moscow, 119991, Russia.

E-mail address: bunkova@mi.ras.ru 